International Journal of Molecular Medicine and Advance Sciences 7 (1): 1-4, 2011

ISSN: $1813-176 \mathrm{X}$

(C) Medwell Journals, 2011

\title{
Physiology of Vitamin D
}

\author{
Stephanie Galea and Renald Blundell \\ Department of Physiology and Biochemistry, University of Malta, Msida, Malta
}

\begin{abstract}
Vitamin D is relevant to several processes in the body mainly in the intracellular mechanisms affecting parathyroid hormone and calcitonin regulation, regulation of vitamin D production by negative feedback and calcium and phosphorous level regulation. Vitamin D may be synthesised in the dermal layer of the skin or absorbed through the diet and utilised. Its biosynthesis initiates from cholesterol and its derivatives and is followed by a cascade of reactions, completed in the kidneys and transported via the blood to where it is utilized. The vitamin D receptor determines the physiological effects of vitamin D and any mutations will cause imbalances in bone homeostasis.
\end{abstract}

Key words: Dihydrocholecalciferol, vitamin D receptor, calcium, parathyroid hormone, phosphate, calcitonin

\section{INTRODUCTION}

Vitamin D is mainly synthesised by the skin when exposed to ultraviolet rays. It can also be obtained through the diet as it is found in oily fish, eggs and fortified cow's milk. The active form of vitamin D binds to Vitamin D Receptors (VDRs) receptors located in bone, the intestines, kidneys, parathyroids and also haematopoietic tissue, cells of the immune system, the prostate in males and several other locations in the body where it helps normal function to occur (Deluca and Cantorna, 2001). Ligand binding to the VDR causes heterodimerisation in conjunction with the retinoic $\mathrm{X}$ receptor in the nucleus to occur, leading to binding of the heterodimer to vitamin D response elements (Costanzo, 2006). This vitamin D receptor complex transcriptionally activates genes including calcium binding genes. The actions of these transcribed genes cause absorption of calcium and phosphate ions. Vitamin $D$ increases calcium entry into cells, movement of calcium through cytoplasm and transfer of calcium into the circulation. Serum calcium levels are regulated by combined action of vitamin D and parathyroid hormone (Bronner and Pansu, 1999).

\section{BIOSYNTHESIS}

Biosynthesis of vitamin D occurs in a series of steps starting from cholesterol being oxidised to provitamin D. Provitamin D is converted to 7-hydrochlesterol by UV light followed by conversion to pre-vitamin $\mathrm{D}$. This is converted by reversible thermal conversion to vitamin $\mathrm{D}_{3}$ in the skin which is also known as cholecalciferol. Mechanisms are present to prevent overproduction of vitamin $\mathrm{D}_{3}$ during overexposure to sunlight since excess vitamin $\mathrm{D}_{3}$ is converted to inactive compounds. Vitamin $\mathrm{D}$ Binding Protein (VDBP) takes up vitamin $\mathrm{D}_{3}$ in the blood to the liver where it is converted to 25hydroxycholecalciferol $\left(25-(\mathrm{OH}) \mathrm{D}_{3}\right)$. This is taken up by the blood and reaches the kidneys where it is converted to 1,25-dihydroxycholecalciferol $\left(1,25-(\mathrm{OH})_{2} \mathrm{D}_{3}\right)$; the active metabolite of vitamin D and 24,25-dihydroxycholecalciferol which is inactive (Costanzo, 2006). Vitamin $D_{2}$ originating from ergosterol in plants, a dietary supplement is metabolised in a similar way (Passeri et al., 2008).

Transcriptional regulation of vitamin $\mathbf{D}_{3}$ : The levels of $25-(\mathrm{OH}) \mathrm{D}_{3}$ are regulated by a negative feedback response which inhibits its production from vitamin $D_{3}$. This is important since the converted form of vitamin $\mathrm{D}$ is not stored and has a half-life of 2 weeks as opposed to the vitamin $\mathrm{D}_{3}$ form which can be stored in the liver for months. Parathyroid Hormone (PTH) controls the production of $1,25-(\mathrm{OH})_{2} \mathrm{D}_{3}$ in the proximal convoluted tubules. The conversion of $25-(\mathrm{OH}) \mathrm{D}_{3}$ to $1,25-(\mathrm{OH})_{2} \mathrm{D}_{3}$ is almost entirely dependent upon PTH. Calcium itself inhibits the formation of $1,25-(\mathrm{OH})_{2} \mathrm{D}_{3}$ and since it suppresses PTH secretion, calcium also causes indirect inhibition of the active metabolite formation. In higher plasma concentrations, calcium also promotes formation of inactive 24,25-dihydroxycholecalciferol which is useful since less Ca uptake from bone, the small intestine and distal renal tubules occurs, bringing serum $\mathrm{Ca}$ back to normal levels around $9.4 \mathrm{mg} \mathrm{dL}^{-1}$ (Guyton and Hall, 2005) (Fig. 1).

The red lines indicate feedback inhibition of parathyroid hormone and $1 \alpha$-hydroxylase whilst the green positive sign and red negative sign show

Corresponding Author: Renald Blundell, Department of Physiology and Biochemistry, University of Malta, Msida, Malta 


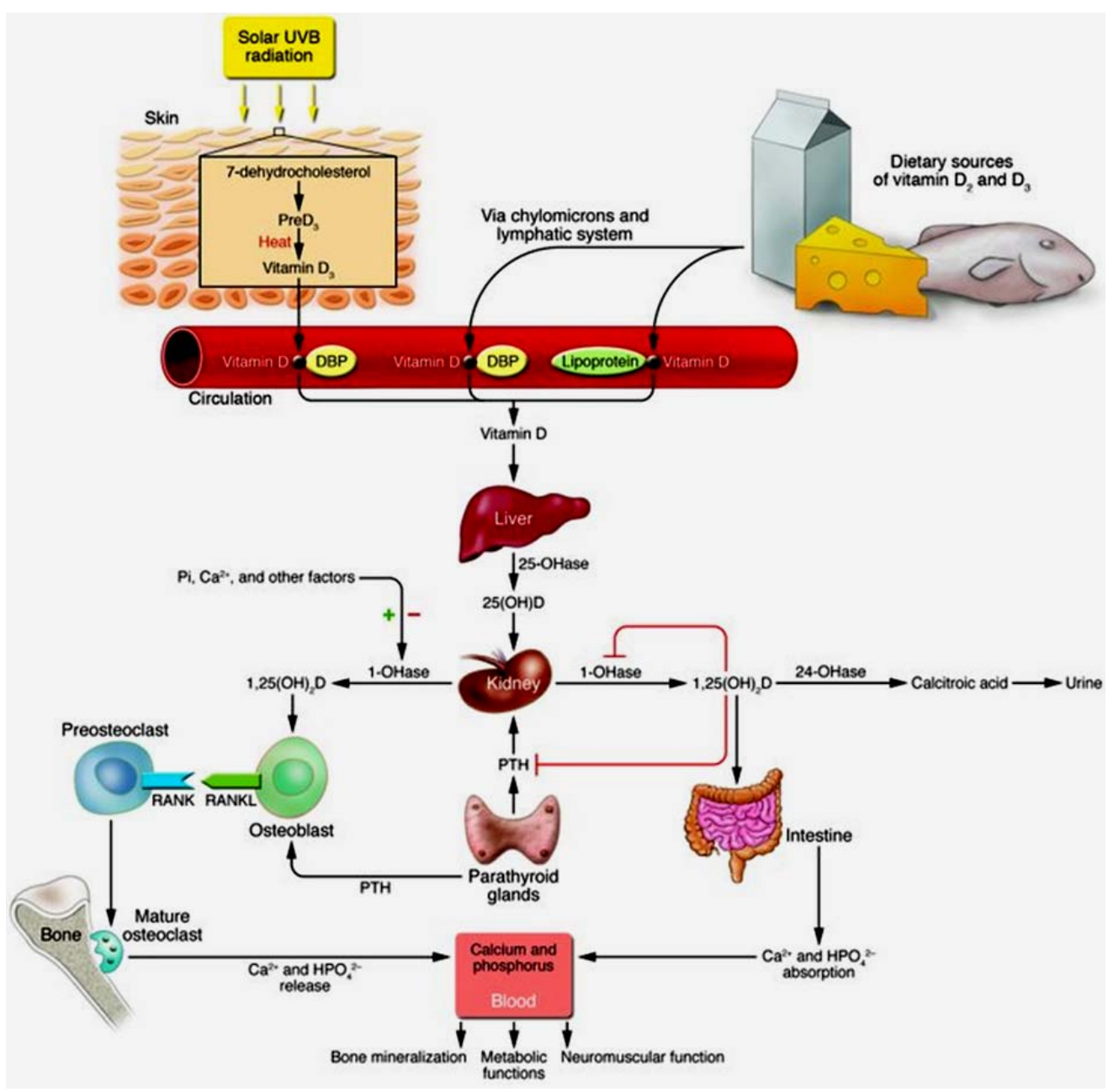

Fig. 1: The sources of vitamin $\mathrm{D}$ in the diet and the main processes of vitamin $\mathrm{D}$ synthesis, metabolism and its effects on the intestine and bone

enhancement and inhibition of $1 \alpha$-hydroxylase. Receptor Activator for Nuclear KB (RANK) stimulates osteoclastic activity in bone (http://www.jci.org/articles/view/29449/ figure/3).

Actions of vitamin D: The actions of vitamin D in its active hormone form are various and more of its importance in the body is being discovered such as in the reproductive especially prostate metabolism and immune system.

Its functions related to bone homeostasis are the following intestinal calcium and phosphate absorption and decreased calcium and phosphate excretion by the kidneys. 1,25- $(\mathrm{OH})_{2} \mathrm{D}_{3}$ increases calcium binding protein, calbindins and calcium channel TRPV6 production in the enterocytes which functions as a transporter for calcium across the cells and into the bloodstream. This protein is stored within cells even if $1,25-(\mathrm{OH})_{2} \mathrm{D}_{3}$ is excreted and so calcium uptake continues. $1,25-(\mathrm{OH})_{2} \mathrm{D}_{3}$ also stimulates the formation of a calcium-stimulated ATPase as well as alkaline phosphatase which seem to promote calcium uptake. When vitamin $\mathrm{D}$ is present in great quantities, it causes absorption of bone by activating osteoblasts which in turn activate osteoclast action. However, in smaller quantities vitamin $\mathrm{D}$ causes bone calcification since, it increases bone mineralisation by modulating calcium influx and increasing calcium binding proteins such as osteocalcin and osteoportin in osteoblasts (Bouillon et al., 2008).

\section{THE VITAMIN D RECEPTOR (VDR)}

VDR expression and its actions in bone: VDR is a receptor expressed by almost all cells in the body and belongs to the steroid receptor family. VDR functions by heterodimerisation together with any of the three isomers 


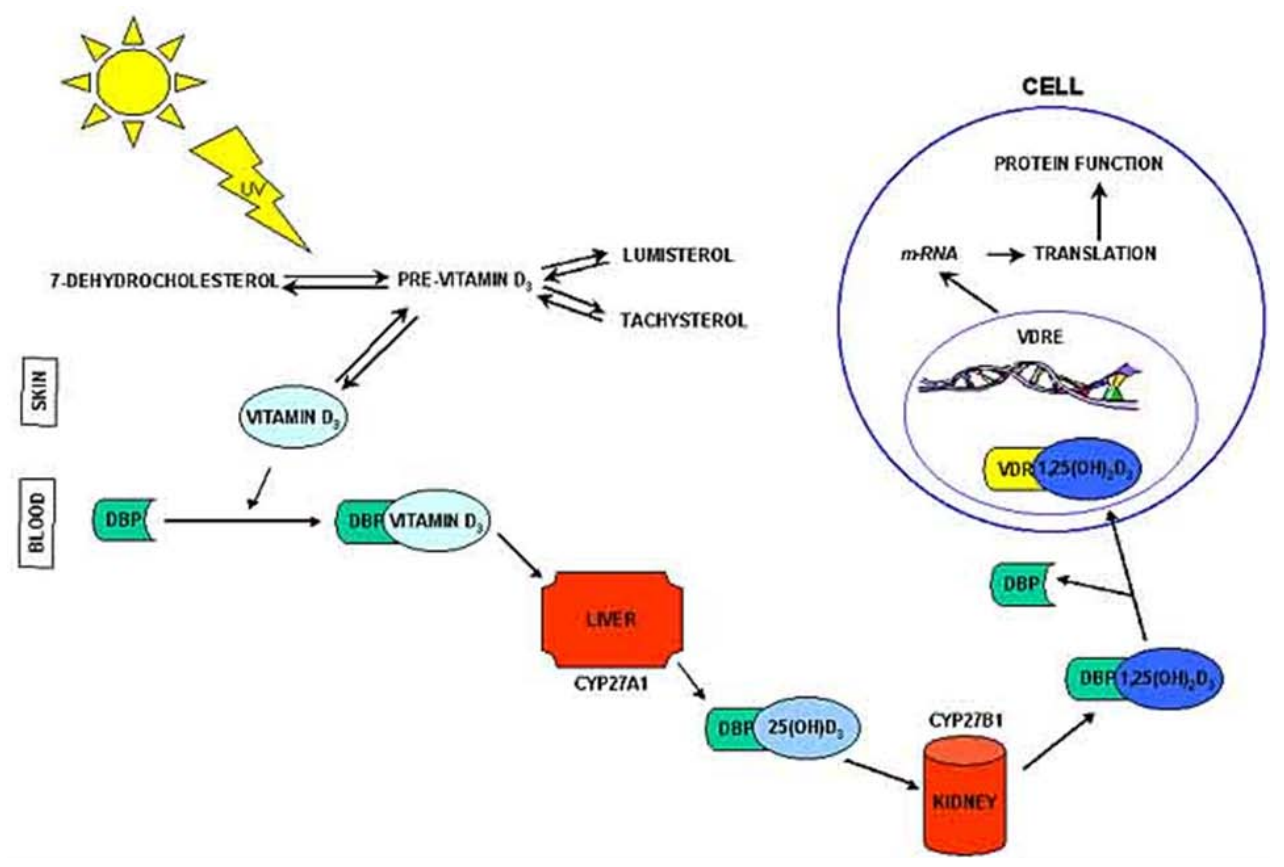

Fig. 2: Vitamin $D_{3}$ synthesis and its transport to target cells

for Retinoid X Receptor (RXR) lacking the specific ligand 9-cis-retinoic acid. When $1,25-(\mathrm{OH})_{2} \mathrm{D}_{3}$ binds to this complex, the active Ligand Binding Domain (LBD) is formed and this complex diffuses through the cell membrane as $1,25-(\mathrm{OH})_{2} \mathrm{D}_{3}$ is a lipid soluble hormone. Once this receptor is activated, a cascade of reactions occur including activation of the MAP-kinase (mitogen activated protein kinase) pathway and an intracellular increase in cyclic Adenosine Monophosphate (cAMP), Protein Kinase C (PKC) and opening of calcium and chloride channels, resulting in a biological response. The main pathway for activation however, is through Vitamin D Response Element (VDRE) activation by binding of the LBD of the VDR to a specific region where zinc fingers seem to play a role of aiding this binding. This modulates gene expression in the nucleus and depending on the cell where it is active, different actions will be brought about. For instance in osteoblasts calcium and chloride channels open which are essential for bone mineralisation and the production of the plasma membrane protein Receptor Activator of NF-kB ligand (RANKL) which promotes osteoclast development. Furthermore in intestinal cells transcaltachia (rapid transport of calcium) and activation of MAP-kinase, PKC and phospholipase C occurs (Norman, 2008).

Polymorphisms of the VDR: A study by Bouillon et al. (2008) was carried out to see the effects of mutations or loss of VDRs in mice. It was found that these mice were suffering from severe hypocalcemia, osteomalacia and rickets as a result of imbalance in calcium and bone homeostasis due to $1,25-(\mathrm{OH})_{2} \mathrm{D}_{3}$ (calcitriol) not being able to act on the receptors. Also, phosphate and PTH balance intestinal absorption and renal calcium reabsorption, seem to be processes dependent on calcitriol activated genes because the mice showed signs of hypophophatemia, secondary hyperparathyroidism, requirement of activation of genes coding for TRPV6, CaBP-9k, PMCA 10 in the intestine and TRPV5 in renal tubular epithelium. These transporters with the exception of TRPV5, do not seem to be entirely dependent on activation by the VDRE complex since the mice did not develop a significant decrease in calcium absorption. This could be due to the presence of other receptors in the enterocytes responsible for calcium uptake.

Figure 2 shows the pathway taken from synthesis of vitamin D in the skin (forming inactive metabolites lumisterol and tachysterol when exposed to excess UV rays) to binding to vitamin $D$ Binding Protein (DBP) and metabolism by cytochrome $\mathrm{P} 450$ oxidase in the liver (encoded by the gene CYP27A1). DBP carries $25-(\mathrm{OH}) \mathrm{D}_{3}$ to the kidneys where another cytochrome P450 oxidase this time encoded by CYP27B1 in the proximal tubules of the kidneys, produces $1,25-(\mathrm{OH})_{2} \mathrm{D}_{3}$. This dissociates from DBP once it reaches the target cell and binds to vitamin D Receptor (VDR). Within the nucleus, binding to 


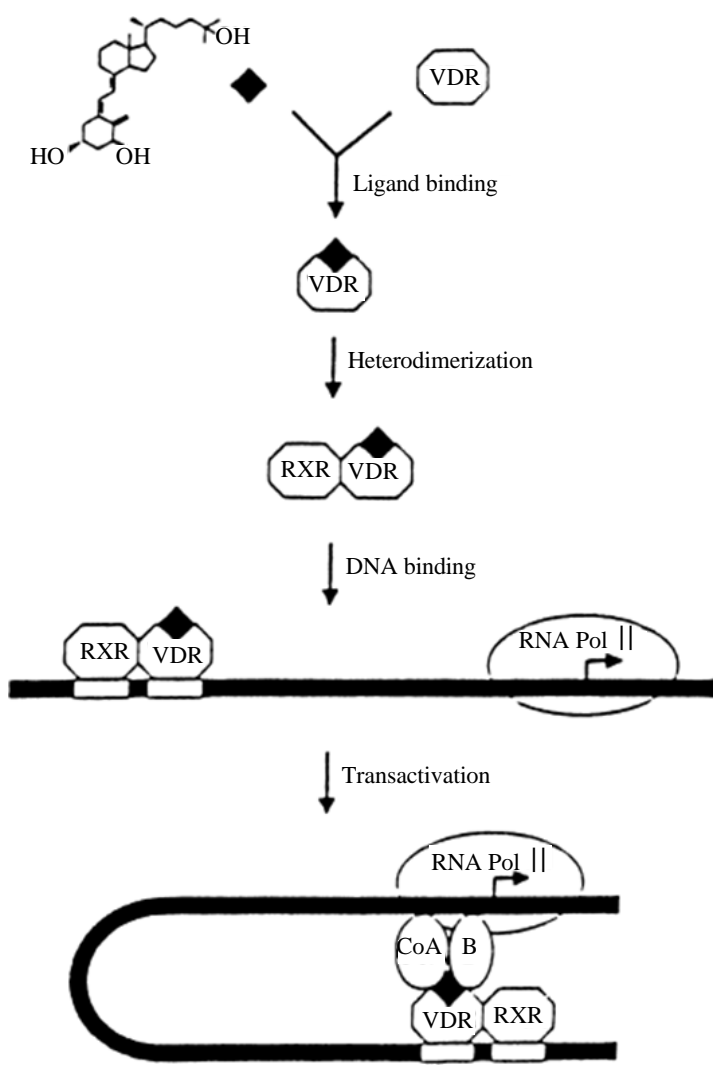

Fig. 3: VDR ligand binding and its intracellular cascade of reactions

the Vitamin D Response Element (VDRE) induces translation of genes to synthesise proteins needed for a particular function (http://www.bioscience.org/2001/v6/d/ hansen/figures.htm).

Binding of 1,25-dihydroxycholecalciferol causes heterodimerization with retinoid X receptor to occur. This complex binds to DNA and after transactivation has occurred, it binds to RNA polymerase 2 as well as coenzyme A (Co A) and transcriptional factor IIB (denoted as B) (Fig. 3).

\section{CONCLUSION}

The study shows that actions of vitamin $\mathrm{D}$ in the body are vast and anything causing an imbalance in this hormone results in consequences such as rickets, osteoporosis and promotion of cancer growth. A decrease in vitamin $\mathrm{D}$ levels may be due to several reasons including polymorphisms of the VDR as well as mutations in the enzymes involved in its biosynthesis. Vitamin D is involved in calcium and phosphate absorption and so these are essential to bone growth and functions.

Its interaction with parathyroid hormone and calcitonin is evident; consequently allowing tight regulation of calcium and phosphate levels, ensuring that homeostasis within the body is maintained.

\section{REFERENCES}

Bouillon, R., G. Carmeliet, L. Verlinden, E. van Etten and A. Verstuyf et al., 2008. Vitamin D and human health: Lessons from vitamin D receptor null mice. Endocrine Rev., 29: 726-776.

Bronner, F. and D. Pansu, 1999. Nutritional aspects of calcium absorption. J. Nutr., 192: 9-12.

Costanzo, L.S., 2006. Physiology. 3rd Edn., Elsevier, Philadelphia, USA., ISBN: 978-1-4160-2320-3.

Deluca, H.F. and M.T. Cantorna, 2001. Vitamin D: Its role and uses in immunology. FASEB J., 15: 2579-2585.

Guyton, A.C. and J.E. Hall, 2005. Textbook of Medical Physiology. 11th Edn., Elsevier, Philadelphia, USA., ISBN-13: 978-0-7216-0240-0.

Norman, A.W., 2008. $1 \alpha, 25(\mathrm{OH})_{2}$ Vitamin $\mathrm{D}_{3}$ Vitamin D Nuclear Receptor (VDR) and Plasma Vitamin D-Binding Protein (DBP) Structures and Ligand Shape Preferences for Genomic and Rapid Biological Responses. In: Principles of Bone Biology, Vol. 1, 3rdEdn., Bilezikian, J.P., L.G. Raisz and T.J. Martin (Eds.). Academic Press Inc., New York, USA., ISBN-13: 978-0123738844, pp: 750-764.

Passeri, G., R. Vescovini, P. Sansoni, C. Galli, C. Franceschi, M. Passeri and The Italian Multicentric Study on Centenarians (IMUSCE), 2008. Calcium metabolism and vitamin D in the extreme longevity. Exp. Gerontol., 43: 79-87. 